\title{
Ultra high energy cosmic rays: clustering, GUT scale and neutrino masses
}

\author{
Z. Fodor ${ }^{\mathrm{a}}$ \\ ${ }^{a}$ Institute for Theoretical Physics, Eötvös University, Pázmány 1, H-1117 Budapest, Hungary
}

The clustering of ultra high energy (above $5 \cdot 10^{19} \mathrm{eV}$ ) cosmic rays (UHECR) suggests that they might be emitted by compact sources. We present a statistical analysis on the source density based on the multiplicities. The propagation of UHECR protons is studied in detail. The UHECR spectrum is consistent with the decay of GUT scale particles and/or with the Z-burst. The predicted GUT mass is $m_{X}=10^{b} \mathrm{GeV}$, where $b=14.6_{-1.7}^{+1.6}$. Our neutrino mass prediction depends on the origin of the power part of the spectrum: $m_{\nu}=2.75_{-0.97}^{+1.28} \mathrm{eV}$ for halo and $0.26_{-0.14}^{+0.20} \mathrm{eV}$ for extragalactic (EG) origin.

\section{Introduction}

The interaction of protons with the microwave background predicts a drop in the cosmic ray flux above the GZK [1] cutoff $\approx 5 \cdot 10^{19} \mathrm{eV}$. The data shows no such drop. Section 2 studies the propagation and determines the probability $P\left(r, E, E_{c}\right)$ that protons created at distance $r$ with energy $E$ reach earth above a threshold $E_{c}$. Using this $P$ one can give the observed spectrum by one numerical integration for any injection spectrum.

It is an interesting phenomenon that the UHECR events are clustered. Recently, a statistical analysis [2] based on the multiplicities of the clustered events estimated the source density. In Section 3 we extend the above analysis.

In Section 4 we study the scenario that the UHECRs are coming from decaying superheavy particles (SP) and we determine their masses $m_{X}$ by an analysis of the observed UHECR spectrum.

Ultrahigh energy neutrinos (UHE $\nu$ ) scatter on relic neutrinos $(\mathrm{R} \nu)$ producing $\mathrm{Z}$ bosons, which can decay hadronically (Z-burst) [3]. In Section 5 we compare the predicted proton spectrum with observations and determine the mass of the heaviest $\mathrm{R} \nu$ via a maximum likelihood analysis.

The details of the presented results and a more complete reference list can be found in 46 .

\section{Propagation of UHECR protons}

Using pion production as the dominant effect of energy loss for protons at energies $>10^{19} \mathrm{eV}$ ref. [7] calculated $P\left(r, E, E_{c}\right)$ for three threshold energies. We extended the results of [7]. In our Monte-Carlo approach protons are propagated in small steps $(10 \mathrm{kpc})$, and after each step the probabilities of pion production and the energy losses due to pair production, pion production and the adiabatic expansion are calculated. We used the following type of parametrization $P\left(r, E, E_{c}\right)=\exp \left[-a \cdot(r / 1 \mathrm{Mpc})^{b}\right]$. Fig. 11 shows $a\left(E / E_{c}\right)$ and $b\left(E / E_{c}\right)$ for a range of three orders of magnitude and for five different $E_{c}$.

\section{Density of sources}

The arrival directions of the UHECRs measured by experiments show some peculiar clustering: some events are grouped within $\sim 3^{\circ}$, the typical angular resolution of an experiment. Above $4 \cdot 10^{19} \mathrm{eV} 92$ cosmic ray events were detected, including 7 doublets and 2 triplets. Above $10^{20} \mathrm{eV}$ one doublet out of 14 events were found [8]. The chance probability of such a clustering from uniform distribution is rather small [8, 8 ].

The clustered features of the events initiated an interesting statistical analysis assuming compact UHECR sources [2]. The authors found a large number, $\sim 400$ for the number of sources within the GZK sphere. We generalize their analysis. The most probable value for the source density is really large; however, the statistical significance of this result is rather weak.

Fig. 2 shows the CL regions for one of our models (injected energy distribution $c(E) \propto E^{-3}$; 


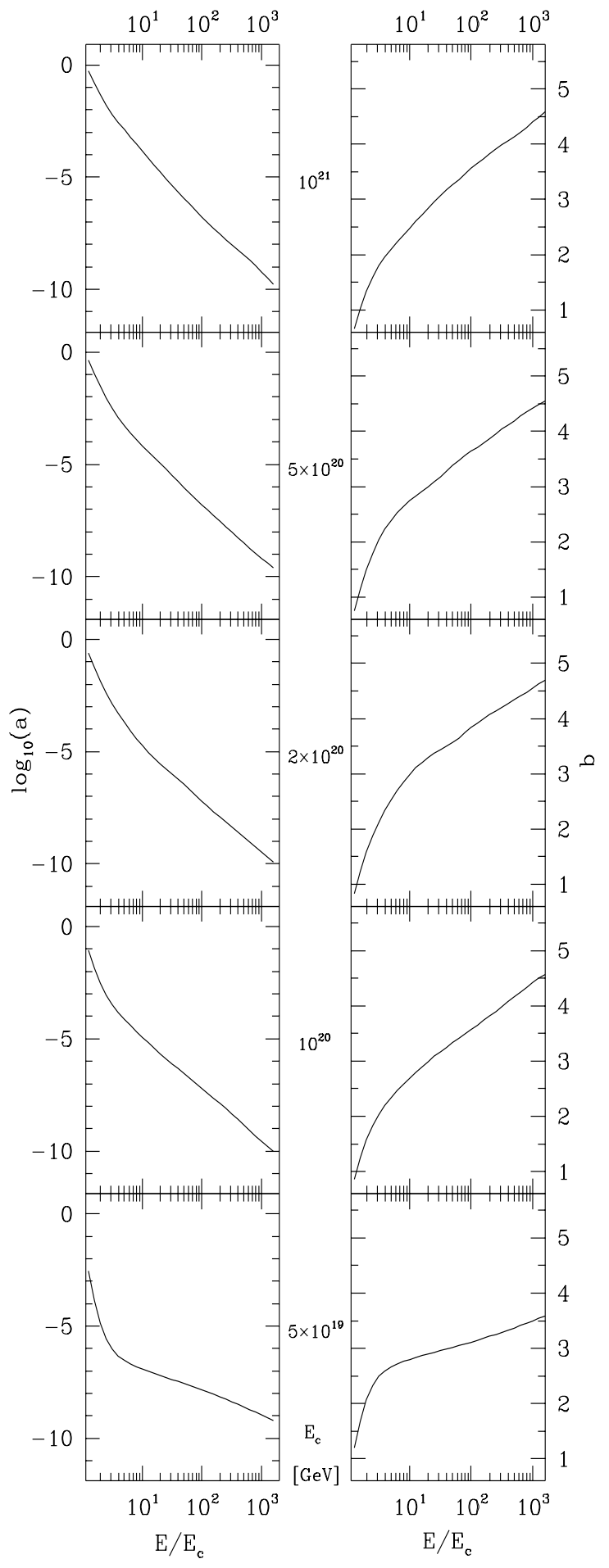

Figure 1. The parametrization of $P\left(r, E, E_{c}\right)$.

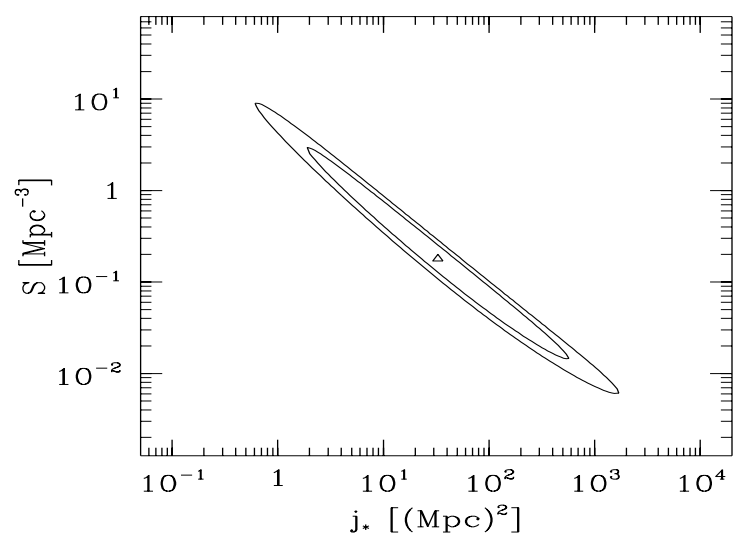

Figure 2. The $1 \sigma(68 \%)$ and $2 \sigma(95 \%)$ confidence level (CL) regions for $j_{*}$ and the source density (14 UHECR with one doublet).

Schechter's luminosity distribution: $h(j) d j \propto$ $\left.\left(j / j_{*}\right)^{-1.25} \exp \left(-j / j_{*}\right) d\left(j / j_{*}\right)\right)$. The regions are deformed, thin ellipse-like objects. For this model our final answer for the density is $180_{-165(174)}^{+2730(8817)}$. $10^{-3} \mathrm{Mpc}^{-3}$, where the first errors indicate the $68 \%$, the second ones in the parenthesis the $95 \%$ CLs, respectively. The choice of [2] $-\mathrm{h}(\mathrm{j}) \propto \delta(\mathrm{j})-$ and, e.g. $E^{-2}$ energy distribution gives much smaller value: $2.77_{-2.53(2.70)}^{+96.1(916)} 10^{-3} \mathrm{Mpc}^{-3}$, which is in a quite good agreement with their result.

\section{Decay of GUT scale particles}

An interesting idea discussed by refs. [10 12] is that SPs could be the source of UHECRs. The hadronic decay of SPs yields protons. They are characterized by the fragmentation function $(\mathrm{FF})$ $D\left(x, Q^{2}\right)$ which gives the number of produced protons with momentum fraction $x$ at energy scale $Q$. For the proton's FF at present accelerator energies we use ref. [13]. We evolve the FFs in ordinary and in supersymmetric QCD to the energies of the SPs. This result can be combined with the prediction of the MLLA technique, which gives the initial spectrum of UHECRs at the energy $m_{X}$ (cf. Fig.. 3). Similar results are obtained by [14].

Depending on the location of the source-halo 


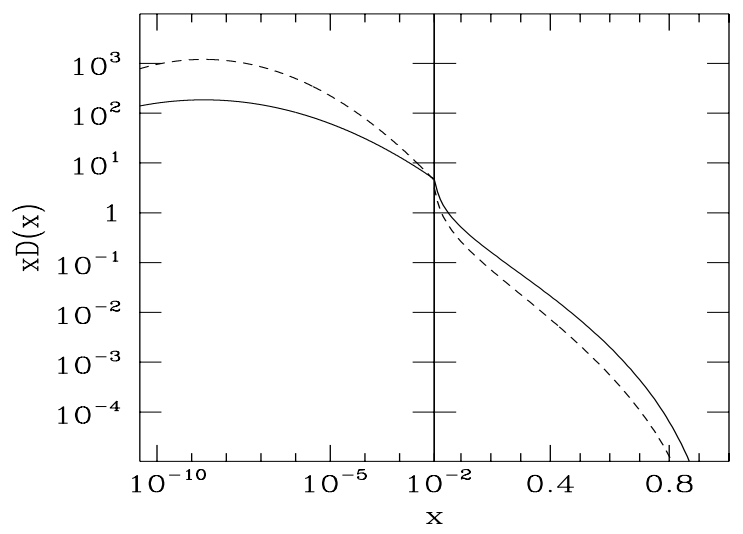

Figure 3. The quark $\mathrm{FFs}$ at $\mathrm{Q}=10^{16} \mathrm{GeV}$ for proton/pion in SM (solid/dotted line) and in MSSM (dashed/dashed-dotted line). We change from logarithmic scale to linear at $x=0.01$.

or extragalactic (EG)- and the model $-\mathrm{SM}$ or MSSM- we study four different scenarios. In the EG case protons loose some fraction of their energies, described by $P\left(r, E, E_{c}\right)$. We compare the predicted and the observed spectrums by a maximum likelihood analysis. This analysis gives the mass of the SP and the error on it.

Fig. 1 shows the measured UHECR spectrum and the best fit, which is obtained in the EGMSSM scenario. The goodnesses of the fits for the halo models are far worse. The SM and MSSM cases do not differ significantly. The most important message is that the masses of the best fits (EG cases) are compatible within the error bars with the MSSM gauge coupling unification GUT scale: $m_{X}=10^{b} \mathrm{GeV}$, where $b=14.6_{-1.7}^{+1.6}$.

\section{Z-burst scenario}

Already in the early 80's there were discussions that the UHE $\nu$ spectrum could have absorption dips at energies around $E_{\nu_{i}}^{\text {res }}=M_{Z}^{2} /\left(2 m_{\nu_{i}}\right)=4.2$. $10^{21}\left(1 \mathrm{eV} / m_{\nu_{i}}\right) \mathrm{eV}$ due to resonant annihilation with R $\nu_{\mathrm{s}}$ of mass $m_{\nu}$, predicted by the hot Big Bang, into $\mathrm{Z}$ bosons of mass $M_{Z}$ [15,16]. Recently it was realized that the same annihilation mechanism gives a possible solution to the GZK problem [3]. It was argued that the UHECRs above

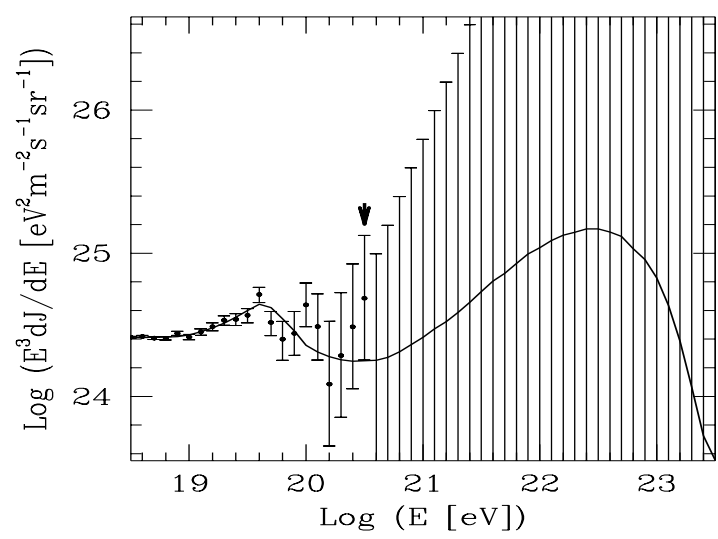

Figure 4. UHECR data with their error bars and the best fit from a decaying SP. There are no events above $3 \times 10^{20} \mathrm{eV}$ (shown by an arrow). Zero event does not mean zero flux, but an upper bound for the flux. thus, the experimental flux is in the "hatched" region with $68 \%$ CL.

the GZK cutoff are from these Z-bursts.

We compare this scenario with observations.

The density distribution of $\mathrm{R} \nu \mathrm{s}$ as hot $\mathrm{DM}$ follows the total mass distribution; however, it is less clustered. Thus we, as opposed to practically all previous authors [3, 17, 19], do not follow the unnatural assumption of having a relative overdensity of $10^{2} \div 10^{4}$ in our neighborhood (for an approach with lepton asymmetry see [18]).

We give the energy distribution of the produced protons in our lab system, which is obtained by Lorentz transforming the CM collider results.

The next ingredient is the propagation of the protons, which can be described by $P\left(r, E_{p}, E\right)$.

Finally, we compare the predicted and observed spectrum and extract the mass of the $\mathrm{R} \nu$ and the necessary UHE $\nu$ flux by a maximum likelihood analysis. In the Z-burst scenario small $\mathrm{R} \nu$ mass needs large $E_{\nu}^{\text {res }}$ to produce a Z. Large $E_{\nu}^{\text {res }}$ results in a large Lorentz boost, thus large proton energy. In this way the detected energy determines the mass of the $\mathrm{R} \nu$. The analysis is completely analogous to that of the previous section.

Our best fits to the observed data gives for the neutrino mass $2.75_{-0.97(1.89)}^{+1.28(3.15)} \mathrm{eV}$ for the "halo"- 


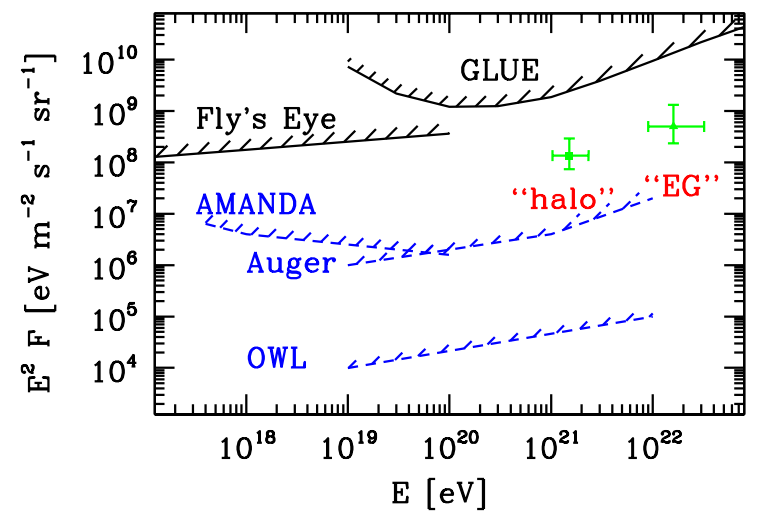

Figure 5. Differential $\nu+\bar{\nu}$ fluxes (averaged over the families) required by the Z-burst hypothesis. The horizontal errors indicate the uncertainty of the mass determination and the vertical errors include also the uncertainty of the Hubble expansion rate. Also shown are upper limits from Fly's Eye and the GLUE, as well as projected sensitivities of AMANDA, Auger and OWL.

and $0.26_{-0.14(0.22)}^{+0.20(0.50)} \mathrm{eV}$ for the "EG"-case, respectively (in the halo/EG cases the power part of the UHECR spectrum is generated in the halo/EG). This gives an absolute lower bound on the mass of the heaviest $\nu$ of $0.06 \mathrm{eV}$ at the $95 \% \mathrm{CL}$.

The most attractive pattern for $\nu$ masses is hierarchical. Using the mass difference of the atmospheric $\nu$ oscillation for the heaviest mass [20], one obtains values between 0.03 and $0.09 \mathrm{eV}$. It is an intriguing feature of our result that the smaller one of the predicted masses is compatible on the $\approx 1.3 \sigma$ level with this scenario.

The necessary UHE $\nu$ flux can be obtained from our fits. We summarized them in Fig. 5 , together with some upper limits. We also compared our $\gamma$-flux with the EGRET bound [21]. Our $\gamma$ flux is somewhat smaller than that of EGRET.

The nice collaboration with S.D. Katz and A. Ringwald and the careful reading of the manuscript is acknowledged. This work was partially supported by Hung. Sci. grants No. OTKA-T34980/T29803/T22929/M28413/OM-MU-708/IKTA111/NIIF.

\section{REFERENCES}

1. K. Greisen, Phys. Rev. Lett. 16, 748 (1966); G.T. Zatsepin and V.A. Kuzmin, Pisma Zh. Exp. Teor. Fiz. 4, 114 (1966).

2. S.L. Dubovsky, P.G. Tinyakov and I.I. Tkachev, Phys. Rev. Lett. 85 (2000) 1154.

3. D. Fargion, B. Mele, A. Salis, Astrophys. J. 517 (1999) 725; T.J. Weiler, Astropart. Phys. 11 (1999) 303; 12 (2000) 379 (Erratum). See also e.g.: T.J. Weiler, hep-ph/0103023; D. Fargion et al., hep-ph/0112014.

4. Z. Fodor, S.D. Katz, Phys. Rev. D 63 (2001) 023002; hep-ph/0105347.

5. Z. Fodor, S.D. Katz, Phys. Rev. Lett. 86 (2001) 3224; hep-ph/0105348.

6. Z. Fodor, S.D. Katz, and A. Ringwald, hepph/0105064; hep-ph/0105336; A. Ringwald, hep-ph/0111112.

7. J.N. Bahcall and E. Waxman, Astrophys. J. 542 (2000) 543.

8. Y.Uchihori et al., Astropart. Phys. 13 (2000) 151.

9. N. Hayashida et al., Phys. Rev. Lett. 77 (1996) 1000.

10. V. Berezinsky, M. Kachelrieß and A. Vilenkin, Phys. Rev. Lett. 79 (1997) 4302.

11. V.A. Kuzmin, V.A. Rubakov, Phys. Atom. Nucl. 61 (1998) 1028.

12. M. Birkel and S. Sarkar, Astropart. Phys. 9 (1998) 297; S. Sarkar, hep-ph/0005256.

13. J. Binnenwies, B.A. Kniehl, G. Kramer, Phys. Rev. D52 (1995) 4947;

14. S. Sarkar and R. Toldra, hep-ph/0108098; R. Toldra, hep-ph/0108127.

15. T.J. Weiler, Phys. Rev. Lett. 49 (1982) 234; E. Roulet, Phys. Rev. D 47 (1993) 5247.

16. S.Yoshida et al., Astrophys. J. 479 (1997) 547.

17. S. Yoshida, G. Sigl, and S. Lee, Phys. Rev. Lett. 81 (1998) 5505.

18. G. Gelmini and A. Kusenko, Phys. Rev. Lett. 82, 5202 (1999).

19. E. Waxman, astro-ph/9804023; J.J. BlancoPillado et al., Phys. Rev. D 61 (2000) 123003.

20. D.Groom et al., Eur. Phys. J. C 15 (2000) 1.

21. P. Sreekumar et al., Astrophys. J. 494 (1998) 523. 